\title{
A BAROMETRIC PRESSURE SENSOR WITH INTEGRATED REFERENCE PRESSURE CONTROL USING LOCALIZED CVD
}

\author{
Patty P. L. Chang-Chien, and Kensall D. Wise
}

\author{
Engineering Research Center for Wireless Integrated MicroSystems \\ Department of Electrical Engineering and Computer Science \\ University of Michigan, Ann Arbor, MI 48109-2122, U.S.A.
}

\begin{abstract}
This paper reports a barometric pressure sensor with integrated reference pressure control. The device was packaged in a cavity, which was created using anodic bonding and subsequently vacuum sealed using localized CVD (Chemical Vapor Deposition) at near ambient temperature. A post-packaging pressure control system, which consisted of in-cavity pressure monitoring and pressure control units, was incorporated with the barometric pressure sensors. The sensor modules, with embedded comb resonators and gettering heaters, were fabricated using a double-polysilicon dissolved-wafer process. The wafers were anodically bonded, thinned, and then released in EDP. Localized CVD was then used to seal the cavity and to react away the residual gas within it using the getters to create the desired reference pressure, as measured using the in-cavity resonators. This implementation required very little extra design space, and only one additional mask compared to the sensor alone. Process improvements have reduced the sealing time and power by a factor of four compared to that previously reported [1]. Sensors capable of resolving $25 \mathrm{mT}$ Torr pressure changes with a sensitivity of $\sim 60 \mathrm{fF} /$ Torr were obtained.
\end{abstract}

Keywords: Hermetic Packaging, Pressure Sensor, Vacuum Control, localized CVD.

\section{INTRODUCTION}

Silicon-based capacitive pressure sensors have been studied extensively in recent years. They are attractive due to their high sensitivity to pressure, low temperature coefficients, and low power consumption compared to their piezoresistive counterparts. Two of the major challenges for absolute pressure sensors are hermetic vacuum sealing and subsequent long-term stability (i.e., outgassing) into the cavity. Both of these lead to a change of pressure in the reference cavity, significantly degrading the sensor accuracy. Accuracy in the absolute pressure sensor cannot be achieved without a knowledge of the reference pressure.

A batch vacuum-sealed barometric pressure sensor capable of resolving 25mTorr over a 300Torr dynamic range [2] has been reported. However, the accuracy of these devices was limited by outgassing into the reference cavity. To improve sensor accuracy, both for as-fabricated and for long-term in-field use, titanium passive getters used for oxygen gettering had been implemented with the absolute pressure sensors [2]. Cavity pressures from 500 mTorr to 10Torr were observed for sensors packaged in a submTorr environment. Corman [3] reported similar results from cavities in which neither gettering nor gas evacuation were implemented. Pressures on the order of 1mbar ( 1.3Torr) were measured from cavities anodically bonded at a background pressure of $10^{-5} \mathrm{mbar}\left(1.33 \times 10^{-2} \mathrm{mTorr}\right)$. Despite that the residual pressure in the sealed environment depends on the amount of gas generated in a given cavity volume, these results suggest that the implementation of a passive getter (such as titanium film) alone is not sufficient. In addition, a minor drift in sensor output (a possible indication of vacuum degradation in the cavity) had also been observed in the absolute barometric pressure sensors [4]. This indicates that an active in-cavity pressure control is necessary for high performance, high-accuracy applications.

We recently demonstrated that localized-CVD can be used to seal wafer cavities at low overall temperature, achieving residual pressures less than 50mTorr as measured with an in-cavity microresonator [1]. This paper significantly extends this previous work to report the first pressure sensor with integrated vacuum sensing/control capability and a localized-CVD seal.

\section{PACKAGING APPROACH}

A post-packaging pressure control system has been designed and demonstrated previously [1]. The general approach is to create the vacuum cavity using an anodic bond, to seal the cavity in a low-pressure CVD environment, and to subsequently monitor the internal cavity pressure and maintain it below a critical level with an active getter. Thus, the three main system components are the first-level package, a pressure-monitoring unit, and an in-chamber pressure control unit. All of the components in this system can be integrated at wafer-level. This produces a significant cost advantage over many active pressure control systems. In addition, an accurate reading of the pressure inside the cavity is not required as long as the pressure is below a critical level. For highperformance pressure sensors and resonators, this critical level is on the order of a few tens of mTorr.

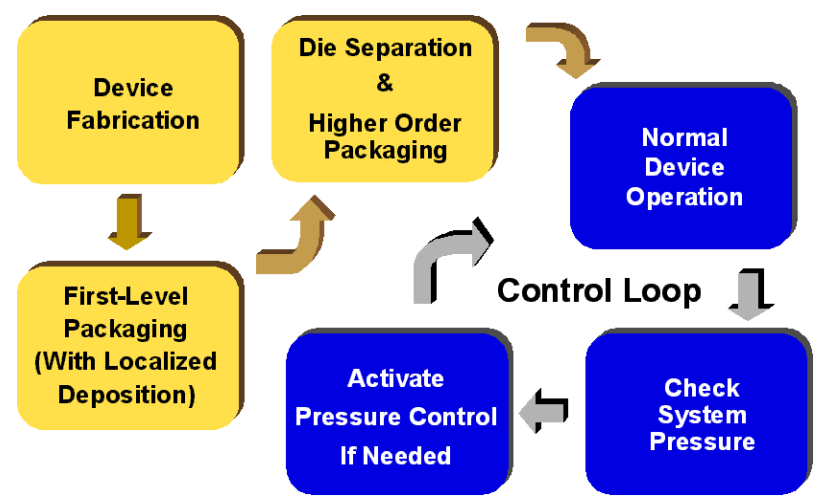

Fig. 1: Post-packaging pressure control system flow chart.

The system flow chart for an active post-packaging pressure control module is shown in Figure 1. After fabrication, waferlevel packaging by localized deposition is performed, followed by 
any higher-level packaging as required. Once packaged, the device operates in its normal mode and cavity pressure is monitored periodically. In the event of vacuum degradation higher than some desired level, pressure control is activated to restore the vacuum back to an acceptable level.

A wafer-level packaging technique that combines anodic bonding and localized heating [1] is utilized to provide a low and predictable pressure in the packaged cavity. The cavity is formed by a recess in the silicon substrate, an incomplete polysilicon ring, and a glass wafer. With this design, oxygen and other gases generated during the bonding process can be pumped out of the cavity through the opening of the polysilicon ring (i.e., the sealing channel). The bonded wafers are then placed in a low-vacuum silane environment to seal the cavity by localized CVD. With this approach, the cavity can be sealed at a controlled pressure near ambient temperature. For simplicity and process compatibility, comb-drive micro-resonators and suspended polysilicon gettering heaters are chosen as the pressure monitoring and pressure control units of the post-packaging pressure control system, respectively.

\section{SYSTEM DESIGN}

The post-packaging pressure control system described above was incorporated into Chavan's barometric pressure sensor design [2]. An optical photograph showing the top view of the pressure sensor module is shown in Figure 2. The reference cavity is formed using an anodic bond between glass and an incomplete polysilicon ring with a sealing heater at the ring opening. The recessed area is composed of a center cavity for the pressure sensor and four side cavities housing two comb resonators and two polysilicon gettering heaters, which are components of the postpackaging pressure control system. The side cavities are linked to the main chamber via connecting channels. The channels are located symmetrically about the main chamber to minimize any geometric imbalance of the diaphragm induced by the openings. The channels are $50 \mu \mathrm{m}$ wide, $45 \mu \mathrm{m}$ long, and $3.5 \mu \mathrm{m}$ tall. The calculated conductance of a channel in $\mathrm{SiH}_{4}$ at room temperature is $5.438 \mathrm{~mm}^{3} / \mathrm{s}$. At this flow rate, the side cavity pressure reaches equilibrium with the main chamber in a fraction of a second.

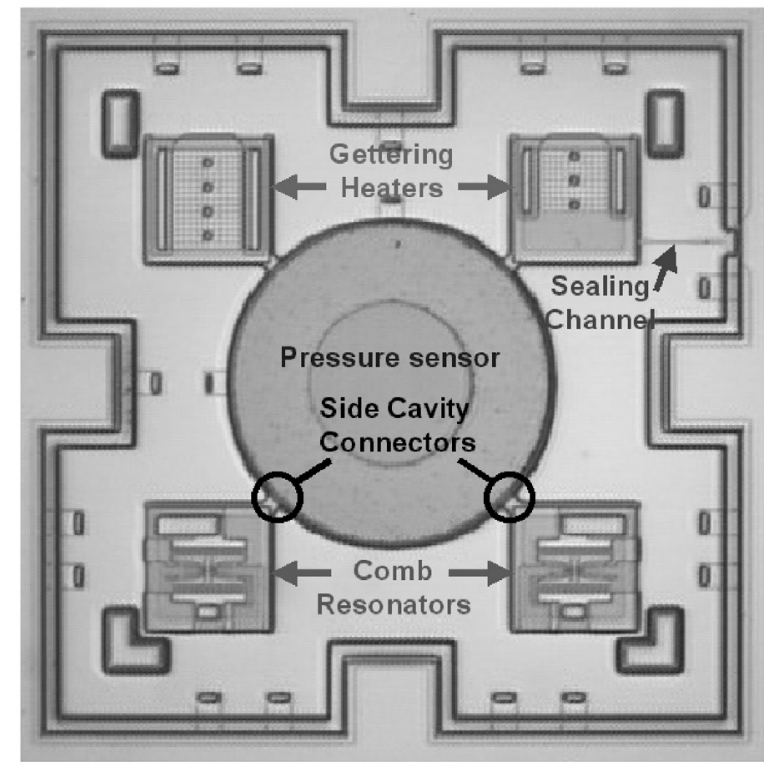

Fig. 2: Optical photograph of a pressure sensor module before anodic bonding showing its system components.
Each side cavity is $420 \mu \mathrm{m}$ by $420 \mu \mathrm{m}$ by $8 \mu \mathrm{m}$. These side cavities take up very little additional chip space since they are located at the four coroners of the circular sensor. It is a great advantage to integrate post-packaging pressure control into the sensor for performance enhancement, with very little overhead cost and only one additional mask compared to the sensor alone. The embedded resonators, which serve as the in-cavity pressure monitoring units, have $2 \mu \mathrm{m}$ wide, $2 \mu \mathrm{m}$ thick, and $20 \mu \mathrm{m}$ long fingers with an overlap of $10 \mu \mathrm{m}$. The spacing between two adjacent fingers was $2 \mu \mathrm{m}$, and the doubly-folded support springs were $50 \mu \mathrm{m}$ long. The gettering heaters, which are the pressure control units, have a resistance of about $250 \Omega$ each. The sealing heater, located at the opening of the sealing channel is $10 \mu \mathrm{m}$ wide and was designed to have a resistance of $\sim 300 \Omega$. The sealing channel shown in Figure 3 is $2 \mu \mathrm{m}$ by $2 \mu \mathrm{m}$ by $125 \mu \mathrm{m}$ at the narrow region. The wider regions, designed to increase the channel conductance, have the dimensions of $11 \mu \mathrm{m}$ by $2 \mu \mathrm{m}$ by $90 \mu \mathrm{m}$, and $11 \mu \mathrm{m}$ by $2 \mu \mathrm{m}$ by $40 \mu \mathrm{m}$. The effective calculated channel conductance is $9.54 \times 10^{-3} \mathrm{~mm}^{3} / \mathrm{s}$. The entire cavity has an approximate volume of $1.12 \times 10^{-2} \mathrm{~mm}^{3}$, which can be filled with $\mathrm{SiH}_{4}$ in $2 \mathrm{sec}$ via the sealing channel.

Pressure sensors with unbossed (regular), bossed, and pedestal-plate diaphragms were implemented. The bossed diaphragm limits the displacement of the sensor and hence improves the linearity at some cost in sensitivity. The sensor with the suspended polysilicon electrode is designed to increase the sensor sensitivity by increasing the effective surface of the sensing area while simultaneously improving linearity.

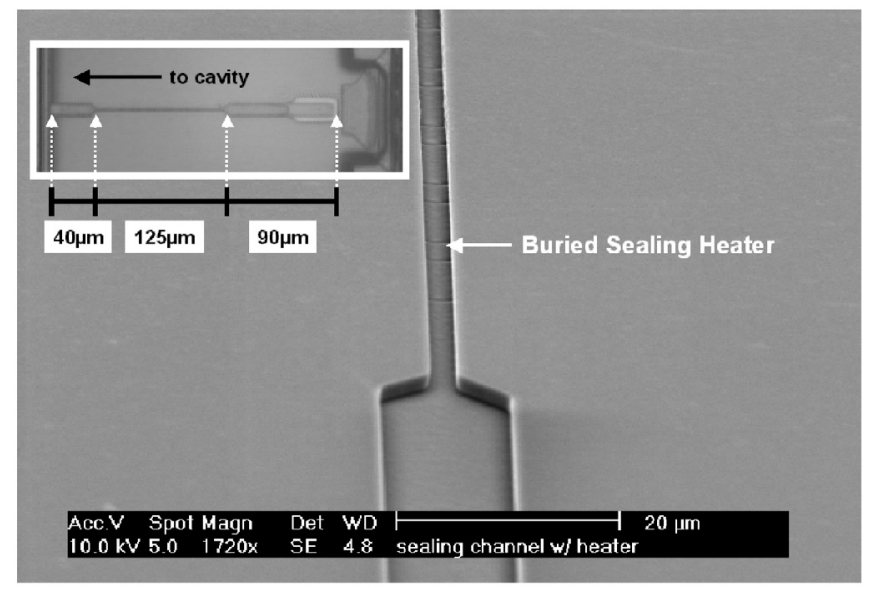

Fig. 3: A SEM showing the sealing channel region, and an insert showing the channel design.

\section{DEVICE FABRICATION}

The silicon wafer was fabricated using a double-polysilicon dissolved-wafer process [2]. Fabrication began with a $7 \mu \mathrm{m} \mathrm{KOH}$ cavity recess followed by $10 \mu \mathrm{m}$ and $4 \mu \mathrm{m}$-deep boron diffusions to form the boss and the diaphragm, respectively. An oxide/nitride layer was deposited for substrate isolation and was patterned to form the poly electrode anchors. The first layer of polysilicon was deposited, doped, and patterned to form device leads, sealing heaters, and electrodes. A second dielectric layer was then patterned to form anchors for the resonators and gettering heaters. Low-stress polysilicon was then deposited and doped, followed by a CMP (Chemical Mechanical Polishing) step. The resonators, heaters, and bonding ring were patterned, followed by an HF 
release. LTO was deposited and patterned, and $\mathrm{Cr} / \mathrm{Au}$ was deposited to form the contacts. The glass wafer was first recessed to form the four side cavities. A layer of $\mathrm{Ti} / \mathrm{Pt} / \mathrm{Au}$ was then deposited to form the electrodes, and the glass was partially diced. Anodic bonding was performed on the two wafers at $400^{\circ} \mathrm{C}$ and 1000V. The Si wafer was then thinned to speed up the release process, and the devices were released in EDP.
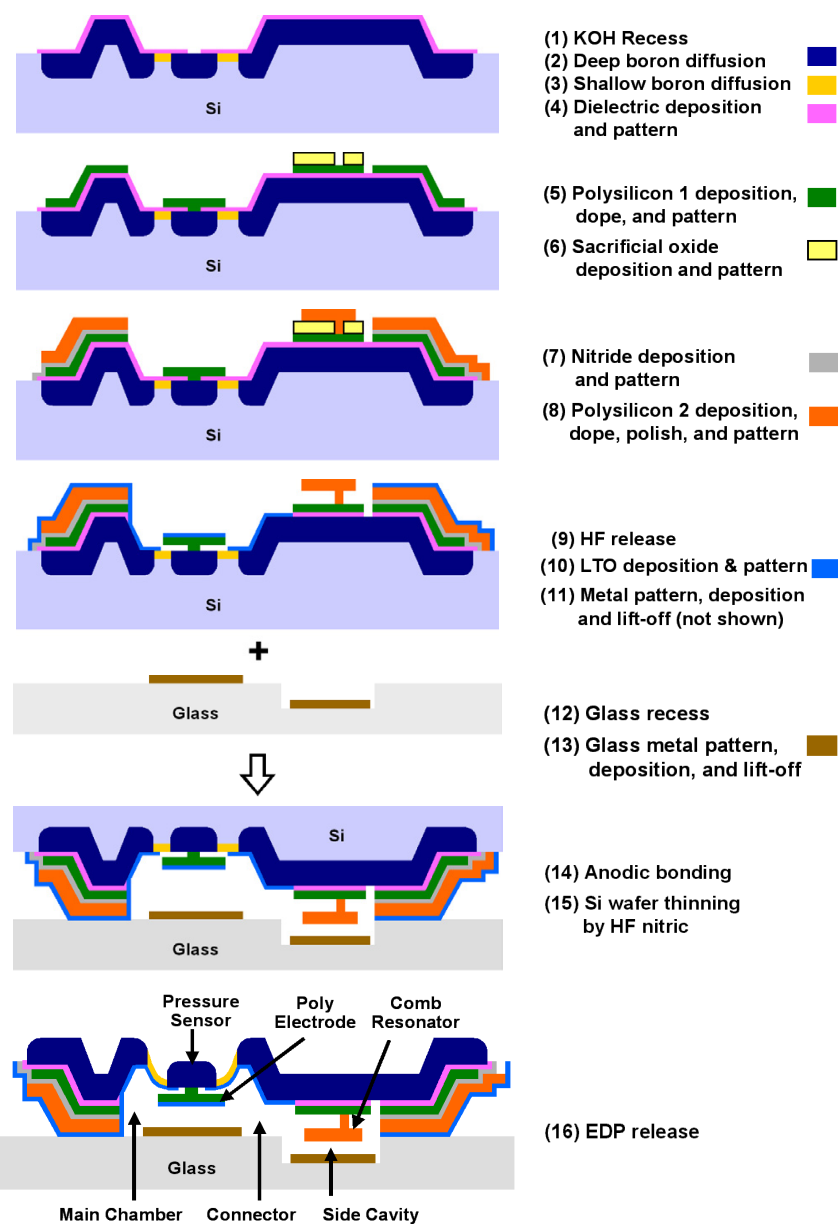
(11) Metal pattern, deposition and lift-off (not shown)

(12) Glass recess

(13) Glass metal pattern deposition, and lift-off

\section{(14) Anodic bonding (15) Si wafer thinning by HF nitric}

(16) EDP release

dimensions, a substantial increase in usable sensing area over any of the bossed diaphragms.

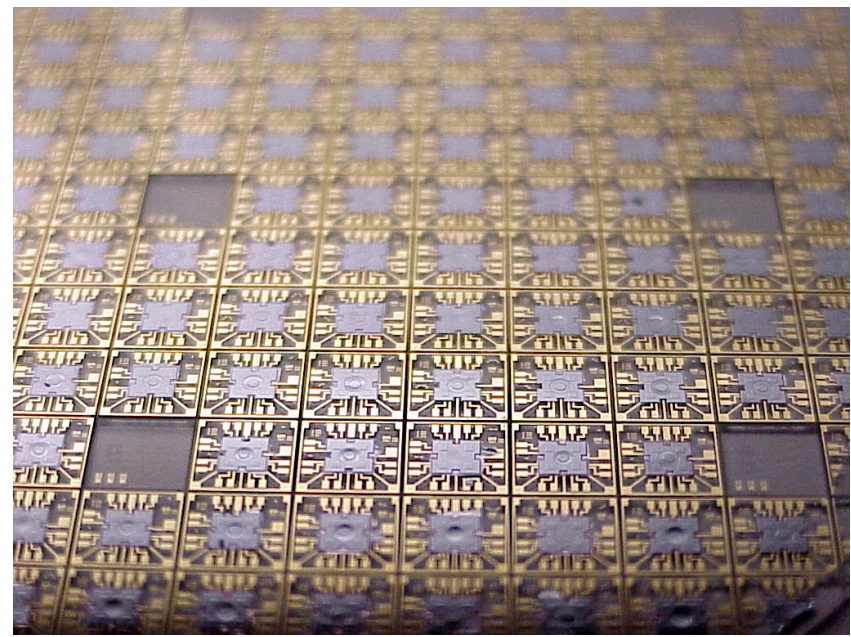

Fig. 5: A fabricated array of barometric pressure sensors.

Fig. 4: Simplified schematic diagram showing the process flow of a barometric pressure sensor with the polysilicon electrode. (Layers not drawn to scale)

\section{EXPERIMENTAL RESULTS}

A fabricated array of the sensor modules is shown in Figure 5. Each device is $5 \mathrm{~mm}$ by $5 \mathrm{~mm}$ on a side. An optical photograph taken from the backside of the module (looking through the glass wafer) is shown in Figure 6. There are total of five different diaphragm dimensions; each is sensitive to a specific pressure range with a total range of 300Torr. The bossed areas for the different boss dimensions are $\sim 0.259 \mathrm{~mm}^{2}, \quad 0.242 \mathrm{~mm}^{2}$, $\sim 0.228 \mathrm{~mm}^{2}, \sim 0.218 \mathrm{~mm}^{2}$, and $\sim 0.203 \mathrm{~mm}^{2}$. Figure 7 is a photograph taken from a module with poly electrode prior to anodic bonding. The individual components are shown in Figures 7 (a) through (e). The poly electrode, as shown in Figure 7 (a), is suspended $2 \mu \mathrm{m}$ above the diaphragm. It is connected physically and electrically to the boss via four small anchors at the center. The small release holes are designed to speed up the release process. The poly electrode has an area of $\sim 0.511 \mathrm{~mm}^{2}$ for all boss

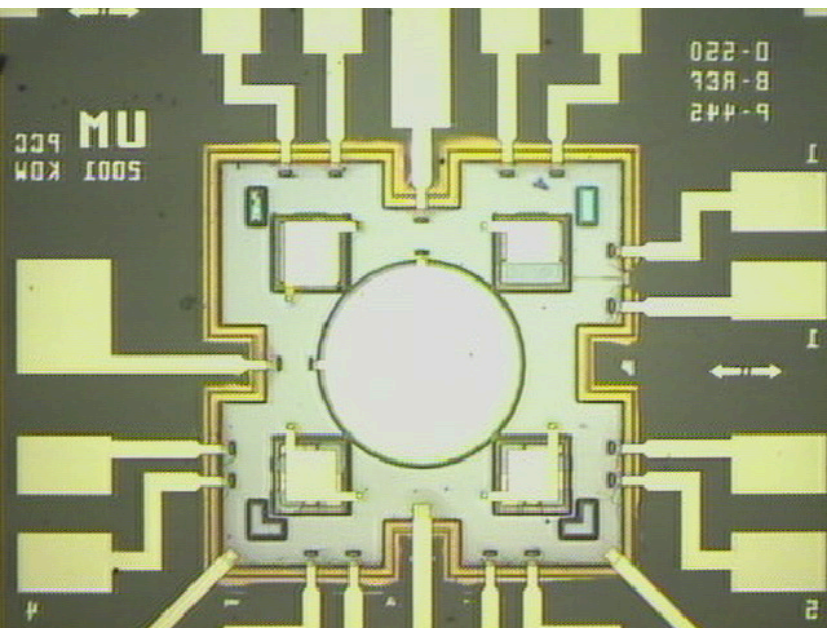

Fig. 6: An optical photograph of the pressure sensor module looking through the glass wafer.

The gettering heaters (Figures $7 \mathrm{~b}, \mathrm{c}$ ) are suspended $2 \mu \mathrm{m}$ above the substrate as well. The large gettering heater has a surface area of $\sim 0.070 \mathrm{~mm}^{2}$, and the small one has an area of $\sim 0.044 \mathrm{~mm}^{2}$. Both heaters are anchored below to small islands of the first layer polysilicon for structur-al support. The small gettering heater is designed such that the path of the sealing channel entrance to the main cavity is cleared of structures in order to ease the gas transport to the other side cavities. The channel has a lid at its opening to seal the entire module during EDP. The channel is later opened by undercutting the lid with an isotropic dry etch.

After fabrication, the sensors are placed in a PECVD chamber for sealing. The individual dies are separated by physically breaking the glass wafer along the pre-diced lines. The dicing lines are only about half-wafer deep so the entire wafer can be handled during the anodic bonding process. The PECVD chamber is used only as a $\mathrm{SiH}_{4}$ supply chamber. The module is sealed by activating the sealing heater. The electrical connections to the heater are provided by an outside power source via electrical feedthroughs. 

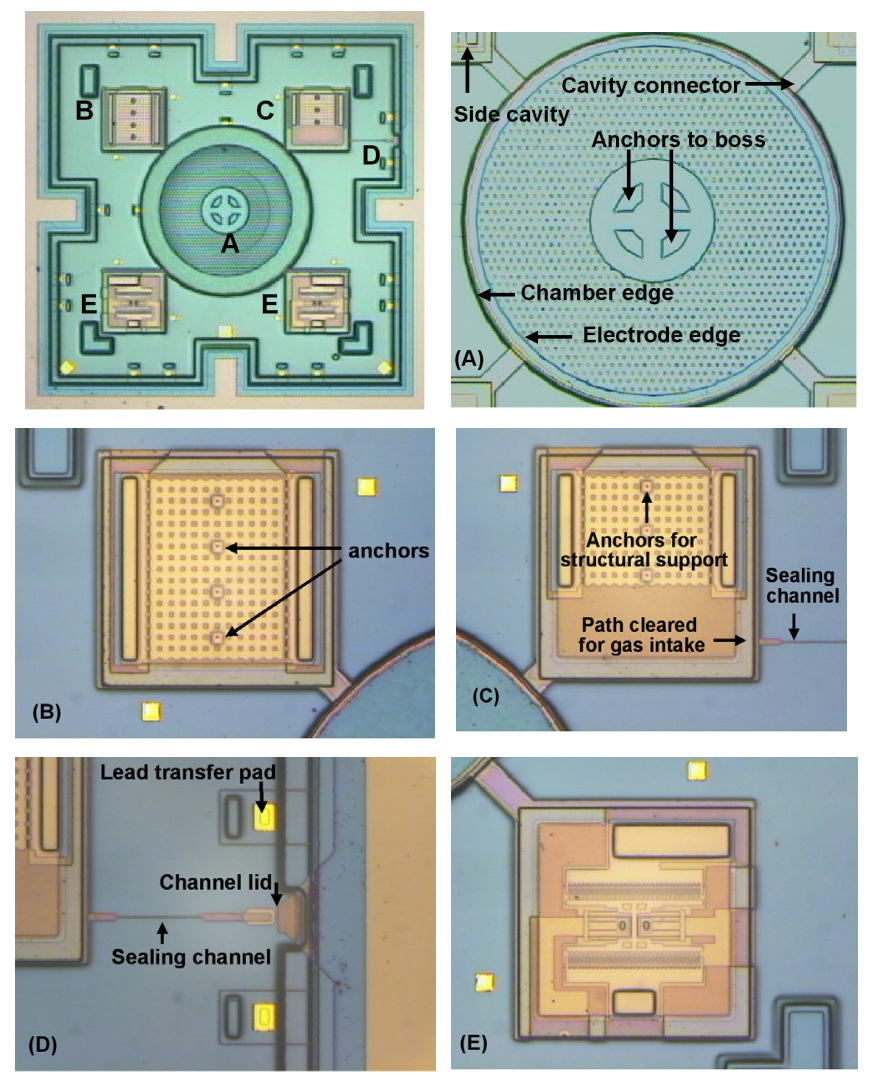

Fig. 7: Optical photographs of a barometric pressure sensor module and its contents. (a) polysilicon electrode, (b) large gettering heater, (c) small gettering heater, (d) sealing channel region, and (e) one of the comb resonators.

The devices are sealed at $200 \mathrm{mT}$ Torr of $\mathrm{SiH}_{4}$ at a flow rate of $80 \mathrm{sccm}$. The performance of the sealing heater was dramatically improved compared to the ones reported previously [1]. The sealing power required was reduced by about a factor of four ( $\sim 620 \mathrm{~mW}$ instead of $\sim 2.2 \mathrm{~W}$ ), and the sealing time was reduced to about 40min for an adequate seal. The estimated local deposition temperature is $600^{\circ} \mathrm{C}$. Figure 8 is an optical photograph of a sealed channel taken through the glass wafer. This improvement in heater performance is a result of process optimization and the removal of the most of the bulk substrate. After sealing, one of the gettering heaters is activated at atmospheric pressure to further reduce the cavity pressure. The estimated sealed pressure after getter activation is 30mTorr.

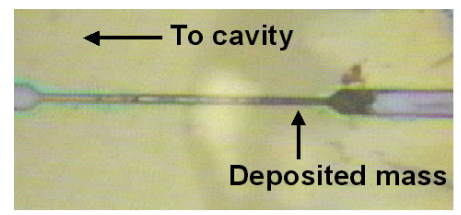

Fig. 8: Optical photograph of a sealed channel (looking through the glass wafer).
The barometric sensors were tested in a pressure chamber and the changes in capacitances were measured as a function of applied pressure. Some of the pressure vs. capacitance characteristics are shown in Figure 9. Sensitivities as high as $~ 60 \mathrm{fF} /$ Torr, which corresponds to a pressure resolution $<25 \mathrm{mT}$, were obtained from the sensors. The measured capacitances of the sensors vary between $8 \mathrm{pF}$ and $11 \mathrm{pF}$ depending on the design.

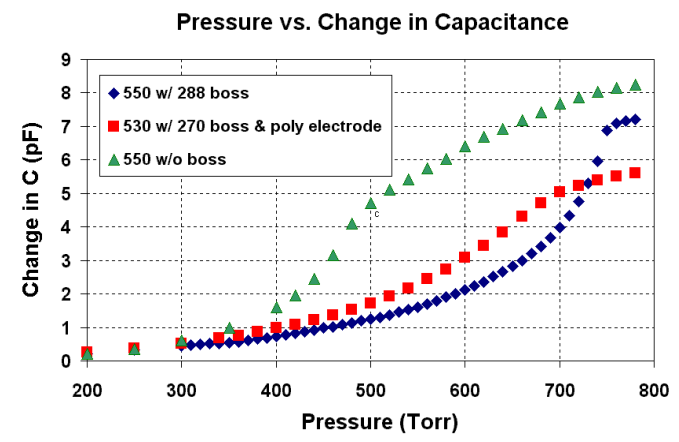

Fig. 9: Pressure vs. change in capacitance data taken from some of the pressure sensors.

\section{CONCLUSIONS}

In this paper, a pressure sensor module with integrated reference pressure control was designed and fabricated. This device was sealed using localized CVD, subsequently reacting any residual gas within the cavity using the getters to decrease the desired reference pressure, as measured using the in-cavity resonators. To incorporate the internal reference cavity control, only one additional mask was necessary compared to the sensor alone. With design and process improvements, the sealing power and sealing time required for the localized CVD were both reduced by about a factor of four compared to that previously reported [1]. The chamber condition during sealing was optimized at a $\mathrm{SiH}_{4}$ pressure of $200 \mathrm{mT}$ Torr at a flow rate of $80 \mathrm{sccm}$. The higher silane flow rate sped up the deposition process and the lower overall pressure ensured a lower after sealing pressure in the cavity. The estimated pressure in the cavity is approximately 30mTorr after gettering heater activation. A sensitivity as high as 60fF/Torr was obtained from a sealed barometric pressure sensor.

\section{ACKNOWLEDGMENTS}

This work is supported by DARPA under the contract number F30602-98-20227, this work made use of ERC shared facilities supported by NSF under award number EEC-0096866.

\section{REFERENCES}

1. P. Chang-Chien, and K. D. Wise, "Wafer-Level Packaging Using Localized Mass Deposition", International Conference on Solid State Sensors and Actuators, Transducers 01, pp. 182-185, 2001.

2. A. V. Chavan and K. D. Wise, "A Multi-Lead Vacuum-Sealed Capacitive Pressure Sensor," Digest Solid-State Sensor and Actuator Workshop, Hilton Head, pp. 212-215, June 1998.

3. T. Corman, P. Enoksson, and G. Stemme, "Low-pressure-encapsulated resonant structures with integrated electrodes for electrostatic excitation and capacitive detection", Sensors and Actuators, A66, pp.16 0-166, 1998.

4. A.V. Chavan, An integrated high resolution barometric pressure sensing system, Doctoral Dissertation, University of Michigan, 2000.

5. H. Henmi, S. Shoji, Y. Shoji, K. Yosimi, and M. Esashi, "Vacuum package for microresonators by glass-silicon anodic bonding", International Conference on Solid State Sensors and Actuators, Transducers 93, pp. 584-587, 1993

6. Y. T. Cheng, L. Lin, and K. Najafi, "Fabrication and hermeticity testing of a glass-silicon packaging formed using localized aluminum/silicon-toglass bonding", International MEMS Conference, pp. 757-762, 2000.

7. H. Guckel, "Surface micromachined pressure transducers", Sensors and Actuators, A28, pp. 133-146, 1991. 